J. Lake Sci. (湖泊科学), $2007,19(1): 46-51$

http:// www. jlakes. org. E-mail: jlakes@ niglas. ac.cn

(c) 2007 by Journal of Lake Sciences

\title{
人工湖滨湿地磷素汇-源功能转换及理论解释”
}

张 奇

(中国科学院南京地理与湖泊研究所,南京 210008)

摘 要: 采用水动力弥散和吸附理论建立了表流湿地水 - 土界面磷通量模型, 定义了湿地汇 - 源转换的临界水体磷浓度

值. 模型揭示, 湿地对磷的截留是水动力弥散和吸附共同作用的结果. 在湿地建成运行初期, 吸附作用明显, 临界浓度值 较低,湿地较好地发挥截磷功能. 随着湿地的运行, 土壤吸附趋于饱和, 吸附作用减弱, 水动力弥散变为主导因素, 临界浓 度值增大,使湿地除磷功能减弱,在一定条件下, 反而可能释放磷. 本文建立的磷通量模型具有明确的物理基础,能解释 湿地对磷去除功能退化的机理和汇 - 源转换条件. 应用模型对抚仙湖马料河湿地的观测数据作了初步解释, 该湿地运行 两年后, 临界磷浓度值为 $0.58 \mathrm{mg} / \mathrm{L}$, 湿地除磷功能明显衰退.

关键词: 人工湿地; 汇 - 源功能; 水动力弥散; 吸附; 磷通量模型

\section{Shift of phosphorus sink-source functions of constructed wetlands and a theoretical explana- tion}

ZHANG Qi

( Nanjing Institute of Geography \& Limnology, CAS, Nanjing 210008,P. R. China)

\begin{abstract}
A mathematical model was presented to calculate phosphorus flux across water-soil interface of constructed wetlands by employing hydrodynamic dispersion and sorption theory. A critical value of inflow phosphorus concentration $\left(C_{R}\right)$ was derived from the model. The critical value can be used to indicate the shift condition as nutrients sink or source of wetlands. According to the model, removal of phosphorus is a joint effect of hydrodynamic dispersion and sorption. During the first few years after construction of wetlands, the sorption process is dominant resulting in a low value of $C_{R}$. During that period, the condition of the wetland as a nutrient sink can be easily satisfied, which make the wetland effectively remove phosphorus. After several years' running, the wetland soil may become sorption-saturated, which make the hydrodynamic dispersion process dominant. This further results in an increased value of $C_{R}$. At that stage, the condition of the wetland as a nutrient sink may be hardly met, and the wetland may function as a nutrient source rather than a sink. The model presented in this paper is physically based and can explain the decline of phosphorus removal efficiency of a wetland with time and the shift condition as a sink or a source. The model was used to interpret the observation data of a constructed wetland right upstream of Fuxianhu Lake, Yunnan Province. It was concluded that the wetland did not have apparent phosphorus removal effects after two years' operating with a value of $0.58 \mathrm{mg} / \mathrm{L}$ for $C_{R}$.
\end{abstract}

Keywords: Constructed wetland; sink-source function; hydrodynamic dispersion; sorption; phosphorus flux model

建造在湖滨带的湿地可以在地表径流人湖前对其携带的污染物质起一定的拦截作用. 随径流进人湿 地的氮、磷物质在一系列物理、化学、生物过程的作用下,被截留在湿地中或转化为气态物质进人大气而离 开径流系统, 使径流在进人湖泊前得到一定程度的净化, 从而减小人湖污染负荷. 人工湖滨湿地作为一种

* 中国科学院“百人计划”项目、国家自然科学基金(40471018)、中国科学院南京地理与湖泊研究所所长基金(CXNIGLAS - 2004-1)、教育部留学回国人员科研基金联合资助. 2006-02-14 收稿;2006-05-29 收修改稿. 张奇, 男,1966 年生,博士,研究员;E-mail : qzhang@ niglas. ac. cn. 
运行成本较低的生态型净水途径,其工作原理正逐渐被人们认识, 并得到广泛的应用 ${ }^{[1-5]}$. 湿地的运行受 水力负荷、污染物负荷、自身内部结构 (土壤、植物)等因素的影响,对污染物的拦截去除效果并不是恒定不 变的,而是处于不断变化之中. Craft 对运行时间小于 15 年的人工湿地与成熟的自然湿地就氮、磷的去除机 理作了对比研究 ${ }^{[6]}$,结果表明,湿地对氮具有可靠的去除效果,这种效果不受湿地运行时间长短和氮负荷 量的影响; 相反, 湿地对磷的拦截效果与湿地运行时间相关性明显, 表现在, 在湿地建成运行的前 $1-3$ 年 中, 磷的去除效果最明显, 因为在这段时间中, 磷的沉淀、吸附过程最显著. 随着湿地运行时间的增长, 土壤 吸附趋于饱和, 磷的截留率明显下降. Craft 的数据显示, 磷的截留率从湿地刚建成后最大 $30 \mathrm{~g} /\left(\mathrm{m}^{2} \cdot \mathrm{a}\right)$ 下 降到湿地运行多年后的 $1-2 \mathrm{~g} /\left(\mathrm{m}^{2} \cdot \mathrm{a}\right)^{[6]}$. Elder 及 Stanley 和 Ward 的研究揭示了湿地对氮、磷去除效果 的不确定性 ${ }^{[7.8]}$, 即所研究的对象有时表现为氮的汇 (去除氮), 有时表现为氮的源 (产出氮), 这种汇 - 源功 能的转换可能发生在同一年内或不同年份间 ${ }^{[8]}$. Elder 的研究还发现,总磷有净的产出,表明所研究的湿地 表现为磷的产出源 ${ }^{[7]}$. 其它研究, 比如, King, Pant 和 Reddy 也表明 ${ }^{[9,10]}$, 湿地受水文条件的影响可能是营 养物的汇, 也可能转变为营养物的源. Richardson 解释说, 淡水湿地生态系统不象陆地生态系统那样可以有 效地截留磷,视湿地土壤特性的不同, 不同湿地对磷的截留容量各不相同 ${ }^{[11]}$. Richardson 进一步指出, 作为 污水处理的湿地在运行几年后将变成 “磷饱和” 状态, 这时, 湿地将不再具有截留磷的功能, 而出现磷 输出 ${ }^{[11]}$.

上述表明,湿地受水文、土壤、污染负荷等因素的影响, 其功能在营养物的汇和源之间存在转换现象. 对主要目的是拦截、去除营养物的人工湖滨湿地来说,湿地的汇 - 源功能转换应引起足够的重视. 特别是, 已有研究表明, 相对于氮,湿地对磷的截留具有更大的不确定性, 湿地也更容易成为磷的产出源区, 而磷又 是湖泊富营养化的极敏感的物质. 因此, 揭示湿地汇 - 源功能转换机理, 探明其转换条件对正确评价湿地 除污效果, 解释湿地运行工况, 改进湿地设计, 科学管理湿地具有重要意义. 本文采用水动力弥散和吸附理 论相结合的新思路, 建立了湿地水 - 土界面磷通量模型, 对湿地汇 - 源功能转换作了理论解释, 并应用模型 对云南抚仙湖北岸的马料河湖滨湿地的运行状况作了分析.

\section{1 水 - 土界面磷通量模型}

Fisher 和 Reddy 阐明,由于湿地土壤是营养物潜在的汇区或源区,研究土壤对营养物质的截留或释放 效应对定量评估土壤对湿地水体中营养物的平衡具有重要意义 ${ }^{[12]}$. Pant 和 Reddy 就湿地土壤中的磷向土 层上部水体的释放作了静态实验研究 ${ }^{[13]}$, 实验发现在第一个水力停留时间 $(28 \mathrm{~d})$ 中,水体中磷的浓度增加 了几倍,表明土层向水体释放了大量的磷. 在随后的几个水力停留时间内,随着水体中磷浓度的增加, 土体 磷释放通量逐渐减小. 该项研究表明, 只有当水中的磷浓度大于等效磷浓度值 (EPCw) 时, 土壤才会对磷起 截留作用;否则, 土壤向水体释放磷,成为磷的内源. 这个结论意味着,当湿地下游的受纳水体的目标磷浓 度小于湿地 $\mathrm{EPCw}$ 时,湿地将不起任何保护下游水体的作用,反而可能会向下游输出磷. 这些研究揭示了湿 地土壤与水体之间存在物质交换,依据水、土的条件,土壤或者是营养物的汇或者是源. 作者认为,湿地水 -土界面上磷通量主要受水动力弥散和吸附这两个过程所控制, 这两个过程的总效果决定了水 - 土界面上 磷通量的强度和方向, 由此构建了磷通量概念模型, 如图 1 示. 由于湿地中水体是流动的, 由此引发的机械 弥散 (mechanical dispersion) 是土层中磷释放的动力之一. 此外, 由于土层与水体间存在磷浓度梯度, 分子 扩散 (molecular diffusion) 也是土层中磷释放的动力. 机械弥散和分子扩散的综合效果称为水动力弥散 (hydrodynamic dispersion), $F_{D}$, 如图 1 示. $F_{D}$ 可表示为:

$$
F_{D}=-D^{*} \cdot \frac{\partial C}{\partial Z}-V \cdot \alpha_{T} \cdot \frac{\partial C}{\partial Z}
$$

式中, $F_{D}$ 为由水动力弥散引起的磷通量 $\left(\left[\mathrm{M} / \mathrm{L}^{2} / \mathrm{T}\right]\right) ; D^{*}$ 为分子扩散系数 $\left(\left[\mathrm{L}^{2} / \mathrm{T}\right]\right) ; V$ 为水流速度 $[\mathrm{L} / \mathrm{T}]$; $\alpha_{\mathrm{T}}$ 为土体横向弥散度 ( $\left.[\mathrm{L}]\right) ; \partial C / \partial Z$ 为水 - 土界面沿 $Z$ 方向的浓度梯度 $\left(\left[\mathrm{M} / \mathrm{L}^{3} / \mathrm{L}\right]\right)$;

式(1)右边第一项表示分子扩散,第二项表示横向机械弥散. 由于水流速度方向与物质弥散方向垂直, 这里采用了横向机械弥散而不考虑沿水流方向的纵向弥散 ${ }^{[14]}$. 合并这两项,式(1) 可改写为:

$$
F_{D}=-\left(D^{*}+V \cdot \alpha_{T}\right) \cdot \frac{\partial C}{\partial Z}
$$




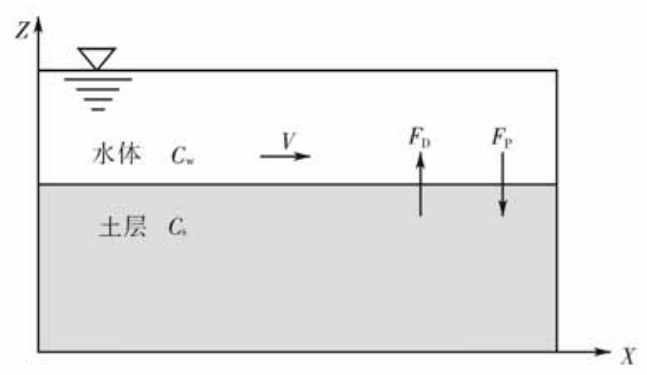

图 1 湿地土壤与水体之间物质交换概念图

Fig. 1 A conceptual model for mass flux across soil-water interface of wetlands

磷的吸附一般采用 Langmuir 模型, 其表达式可 写为 ${ }^{[15]}$ :

$$
F_{P}=-\frac{\alpha \cdot \beta \cdot C_{W}}{1+\alpha \cdot C_{W}}
$$

式中, $F_{P}$ 为吸附通量 $\left(\left[\mathrm{M} / \mathrm{L}^{2} / \mathrm{T}\right]\right) ; \alpha$ 为常数 $\left(\left[\mathrm{L}^{3} / \mathrm{M}\right]\right) ; \beta$ 为可能发生的最大吸附量 $\left(\left[\mathrm{M} / \mathrm{L}^{2} / \mathrm{T}\right]\right) ; C_{W}$ 为水体中磷的 浓度 $\left(\left[\mathrm{M} / \mathrm{L}^{3}\right]\right)$; 负号表示磷从水体进人土体(见图 1 坐标 系).

式(3)表示, 土壤对磷的吸附量与水体中磷的浓度有 关, $C_{W}$ 越大, 吸附量越大, 吸附量的极限值为 $\beta$, 变量间的相 互关系可用图 2 表示. 湿地运行数年后, 土壤吸附将趋于饱 和,因此, $\beta$ 值是随时间变化的, 从湿地初建成的最大值逐渐

变小,当湿地土壤完全饱和后, $\beta$ 值变为 0 .

叠加式(2)和 (3), 并将式 (2) 中的浓度微分项用差分表示, 则某时段内水 - 土界面的物质通量变化 量为:

$$
F=F_{D}+F_{P}=\frac{D^{*}+\alpha_{T} \cdot V}{d} \cdot\left(C_{S}-C_{W}\right)-\frac{\alpha \cdot \beta \cdot C_{W}}{1+\alpha \cdot C_{W}}
$$

式中, $F$ 为水 - 土界面物质通量变化量 $\left(\left[\mathrm{M} / \mathrm{L}^{2} / \mathrm{T}\right]\right) ; d$ 为与水体发生磷交换的土壤层厚度 $([\mathrm{L}]) ; C_{S}$ 为土 壤磷浓度 $\left(\left[\mathrm{M} / \mathrm{L}^{3}\right]\right)$.

式(4)表示,水-土界面磷净通量为水动力弥散和吸附共同作用的结果, 如图 3 示 (取 $C_{S}$ 为 $0.5 \mathrm{mg} /$ $\mathrm{L}$ ). 由图 3 的 $F_{D}$ 曲线知, 当 $C_{W}$ 小于 $C_{S}$ 时, 水动力弥散使土壤释放磷 ( $F_{D}$ 为正值), 反之, 水体中的磷进人 土壤 ( $F_{D}$ 为负值). 叠加吸附曲线 $F_{P}$ 后得到 $F$ 曲线, 该曲线与横坐标的交点数值为 $C_{R}$. 由图 3 知, 只有当 $C_{W}$ 大于浓度值 $C_{R}$ 时, 湿地土壤才具有截留磷的功效 ( $F$ 为负值). 浓度值 $C_{R}$ 定义为湿地土壤汇 - 源功能 转换的临界值. $C_{R}$ 的实践意义在于,在湿地运行中,应保持 $C_{R}$ 值尽可能小,使湿地在大多数条件下都具备 除磷的功能. 然后, 随着湿地土壤趋于磷饱和, 吸附作用逐渐减弱, $F$ 曲线向 $F_{D}$ 曲线演变, $C_{R}$ 值逐渐增大, 湿地除磷功效逐渐减弱.

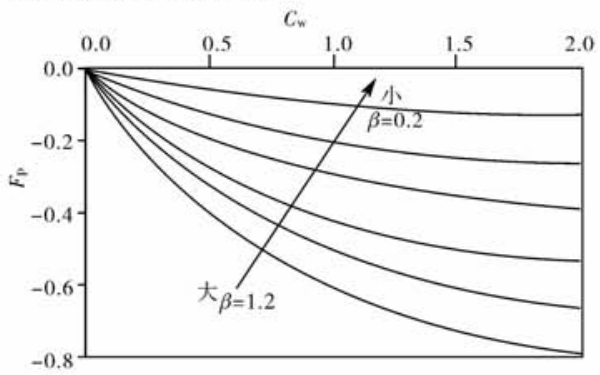

图 2 Langmuir 吸附模型 $F_{P}, C_{W}, \beta$ 间的关系

Fig. 2 Relationship of $F_{P}, C_{W}$ and

$\beta$ for Langmuir sorption model

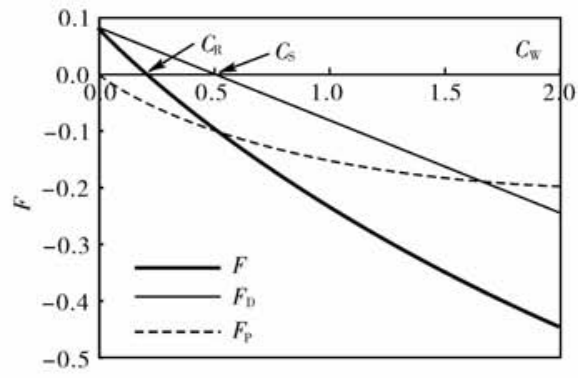

图 3 水动力弥散和吸附对磷通量的影响

Fig. 3 Effects of hydrodynamic dispersion and sorption on phosphorus flux

由式(4) 推得, 当 $C_{W}=0$ 时, $F=\frac{D^{*}+\alpha_{T} \cdot V}{d} \cdot C_{S}$, 该值为由水动力弥散引起的潜在最大磷释放量. 令, $D_{T}^{\prime}=d /\left(D^{*}+\alpha_{T} \cdot V\right), C_{S}{ }^{\prime}=\alpha \cdot C_{S}$, 由式(4) 可得 $\mathrm{C}_{\mathrm{R}}$ 为:

$$
C_{R}=\left[\sqrt{\left(\alpha \cdot \beta \cdot D_{T}^{\prime}+1-C_{S}^{\prime}\right)^{2}+4 C_{S}^{\prime}}-\left(\alpha \cdot \beta \cdot D_{T}^{\prime}+1-C_{S}^{\prime}\right)\right] /(2 \alpha)
$$

\section{2 模型应用}

应用上述模型原理对云南抚仙湖北岸的马料河河口湿地观测数据作分析. 马料河是抚仙湖北岸的主 
要人湖河流之一, 流域面积 $8.01 \mathrm{~km}^{2}$,多年平均降雨量 $1032.7 \mathrm{~mm}$, 多年平均径流量 $3.31 \times 10^{6} \mathrm{~m}^{3}$. 河流流经 农田,主要受农田面源污染. 为了在受污河水人湖之前尽可能地消减污染物,在湖滨带修建了人工湿地. 湿 地由 3 个串联起来的功能区组成:沉淀池、潜流区和表流区,其中表流区的面积最大,为 $11550 \mathrm{~m}^{2}$. 表流区 底板为天然泥土质, 种植水芹菜. 将河水引人湿地, 经湿地再排人湖泊. 湿地于 2003 年 10 月建成并投人运 行. 于 2005 年 6 月 6 日 - 2005 年 9 月 26 日对该湿地作观测. 采样频率为 $1-2 \mathrm{~d}$,分别在表流区进水口和 出水口采样,遇雨天则停止采样. 观测指标有氨氮 $\left(\mathrm{NH}_{4}^{+}\right)$、硝氮 $\left(\mathrm{NO}_{3}^{-}\right)$、总氮 $(\mathrm{TN})$ 、磷酸盐 $\left(\mathrm{PO}_{4}^{3-}\right)$ 和总磷 (TP) ,水质分析方法参考文献[16].
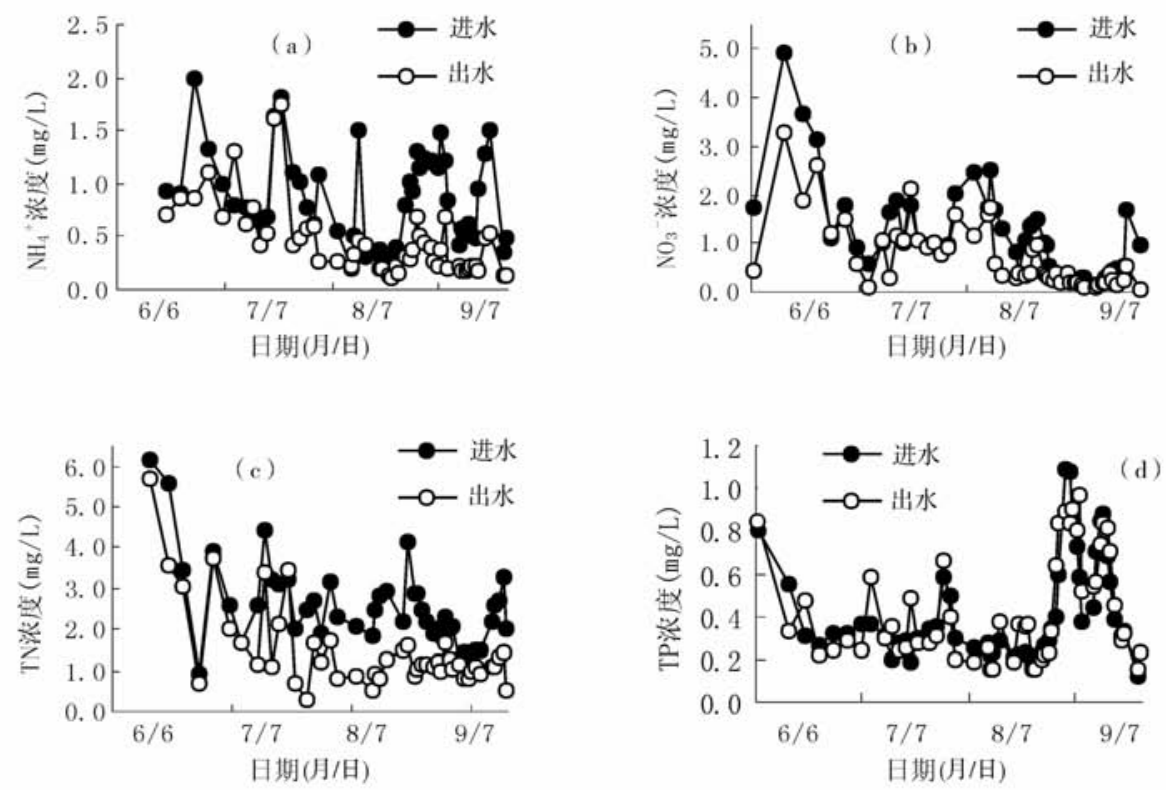

图 4 湿地表流区进出水氮磷浓度比较(2005 年)

(a) $\mathrm{NH}_{4}^{+}$浓度; (b) $\mathrm{NO}_{3}{ }^{-}$浓度; (c) $\mathrm{TN}$ 浓度; (d) $\mathrm{TP}$ 浓度

Fig. 4 Observed inflow and outflow N, P concentrations, 2005

(a) $\mathrm{NH}_{4}^{+}$; (b) $\mathrm{NO}_{3}{ }^{-}$; (c) $\mathrm{TN}$; (d) $\mathrm{TP}$
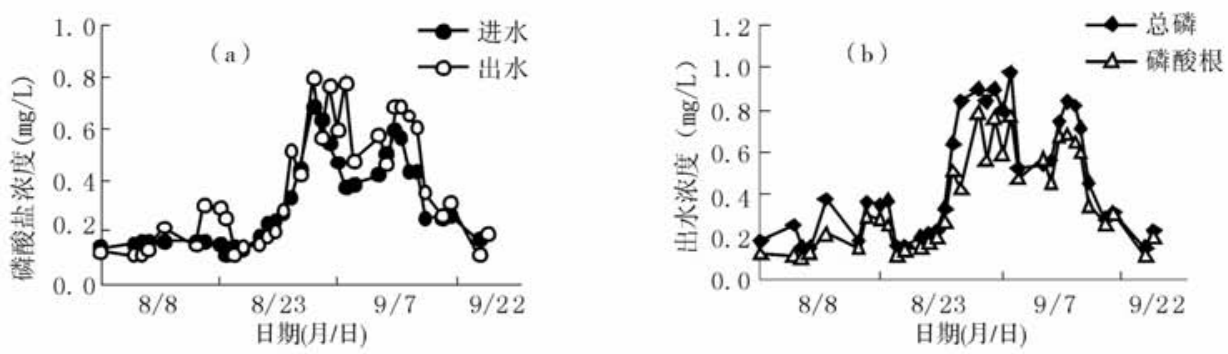

图 5 可溶性磷酸盐浓度变化及在总磷中的含量(2005 年)

(a) 磷酸盐浓度变化; (b) 磷酸根在总磷中的含量

Fig. 5 Concentrations of soluble phosphate in inflow, outflow and total phosphorus

(a) Phosphate concentrations; (b) Phosphate concentration in relative to TP 
图 4 为湿地表流区观测时段内进出水浓度比较. 由图 4(a-c) 可见,出水中氮的浓度低于进水浓度. 表流区没有其它水的人口或出口, 当忽略水面蒸发时, 可以认为进口流量等于出口流量, 所以氮的出口负荷 小于进口负荷, 表明表流区湿地对各形态氮具有较为稳定的去除功能. 湿地中的反硝化是较为稳定的过 程,该过程使硝氮转化成气态物质离开水体,所以,通常湿地对氮具有较为稳定的去除效果. 这个结果与其 他研究是吻合的 ${ }^{[6]}$. 由图 4(d) 可见,出水总磷浓度没有明显下降, 反映表流区对总磷没有明显的去除效 果. 计算表明,进、出水平均总磷浓度分别为 0.410 和 $0.428 \mathrm{mg} / \mathrm{L}$, 表明在观测时段内,表流区总的功能表 现为磷的源区, 对磷没有任何去除功效, 反而, 内部出现磷的释放. 这个结果与 Mitsch 和 Reeder 的研究结果 相似,他们在美国俄亥俄州的一个海岸区淡水湿地观测到的进、出水平均总磷浓度分别是 0.247 和 0.248 $\mathrm{mg} / \mathrm{L}^{[17]}$.

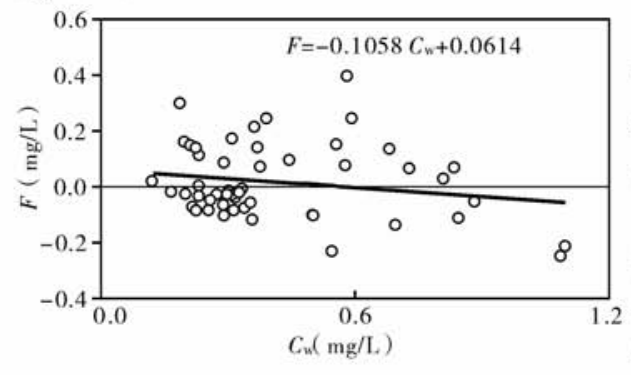

图 6 表流湿地进出水总磷浓度差 与进水总磷浓度的关系

Fig. 6 Correlation of difference of inflow and outflow TP concentrations with inflow TP concentration

对水样的可溶性磷酸盐的分析发现,该湿地可溶性磷酸 根的出水浓度也表现出大于进水浓度的趋势, 如图 5(a) 示. 这个规律与总磷的进出水浓度变化是相一致的. 进一步的 分析知道, 湿地来水中, 总磷的主要成分为磷酸根, 如图 5 (b) 示. 尽管从理论上讲, 基于水动力弥散理论的磷通量模 型式(4)更适合于模拟可溶性磷的迁移, 由于磷酸根含量接 近于总磷浓度, 这里采用时间系列较长的总磷数据作定量分 析. 图 6 为由观测数据得到的湿地表流区进出水磷浓度差与 进水浓度的关系, 负 $F$ 值表示出水浓度小于进水浓度; 正 $F$ 值表示出水浓度大于进水浓度. 图中的粗实线为观测点的 趋势线, 该线相当于图 3 中的 $\mathrm{F}$ 线, 直线的方程见图示. 由趋 势线方程求得 $C_{\mathrm{R}}$ 为 $0.58 \mathrm{mg} / \mathrm{L}$, 即只有当进水浓度 $C_{\mathrm{W}}$ 大于 $0.58 \mathrm{mg} / \mathrm{L}$ 时, 湿地才可能截留部分磷. 在所观测的样本中, 只有 $21 \%$ 点次的浓度大于 $0.58 \mathrm{mg} / \mathrm{L}$, 说明在大多数情况下, 湿地不具备截留磷的功能,湿地运行两年多后, 土壤对磷的吸附可能已趋于饱和. 在获取更多现场参数后, 将可以应用式(4)对该湿地的运行作更为详细的定量分析.

\section{3 结论}

人工湿地去除水中营养物质的效果受水文、污染负荷、湿地本身条件等因素的影响,对不同形态物质具 有不同的去除效果. 已有研究和本文研究表明,湿地对氮具有较为稳定的去除效果, 而对磷表现出不稳定 的去除效果. 本文采用水动力弥散和吸附理论建立了表流湿地水 - 土界面磷通量模型, 并由此引出了湿地 汇 - 源转换的临界磷浓度值. 根据该模型, 湿地对磷的截留是水动力弥散和吸附共同作用的结果. 在湿地 建成运行初期, 吸附作用明显, 临界浓度值较低,湿地能较好发挥去磷功能. 随着湿地的运行, 土壤吸附趋 于饱和, 吸附作用减弱, 水动力弥散开始起主导作用, 使临界浓度值增大, 湿地在大多数情况下不能有效去 除磷, 反而可能释放磷. 本文建立的水 - 土界面磷通量模型具有明确的物理基础, 并能满意解释湿地对磷 去除功能的退化机理和汇 - 源转换条件. 应用该理论对抚仙湖北岸湖滨区湿地的观测数据作了初步分析, 得出该湿地汇 - 源转换的临界磷浓度值为 $0.58 \mathrm{mg} / \mathrm{L}$. 数据显示, 该湿地运行两年后已基本不具备除磷效 果. 在进一步取得现场数据, 并结合室内实验, 将可以对模型作更为详细的参数反演, 使模型对该湿地磷的 迁移作定量模拟和预测.

致谢: 马料河湿地现场观测由研究生徐进、刘佳实施; 观测工作得到了中科院南京地理与湖泊研究所李文朝 研究员和徐力刚助理研究员的支持和帮助.

\section{4 参考文献}

[1] 姜翠玲, 崔广柏. 湿地对农业非点源污染的去除效应. 农业环境保护, 2002, 21(5): 471 -473,476.

[2] 刘文祥. 人工湿地在农业面源污染控制中的应用研究. 环境科学研究, 1997, 10(4): 15-19. 
[3] 许春华, 周 琪, 宋乐平. 人工湿地在农业面源污染控制方面的应用. 重庆环境科学, 2001, 23(3)： $70-72$.

[4] 陈源高, 吴献花, 李文朝, 孔志明. 抚仙湖窑泥沟人工湿地的除磷效果研究. 应用生态学报, 2005, 16(10): $1913-1917$.

[5] Jordan T E, Whigham D F, Hofmockel K H et al. Nutrient and sediment removal by a restored wetland receiving agricultural runoff. Journal of Environmental Quality, 2003, 32: 1534 - 1547.

[6] Craft C B. Dynamics of nitrogen and phosphorus retention during wetland ecosystem succession. Wetlands Ecology and Management, 1996, 4(3): 177 - 187.

[7] Elder J F. Nitrogen and phosphorus speciation and flux in a large Florida river wetland system. Water Resources Research, 1985, 21(5): $724-732$.

[8] Stanley E H, Ward A K. Inorganic nitrogen regimes in an Alabama wetland. Journal of the North American Benthological Society, 1997, 16(4): $820-832$.

[9] King D L. Nutrient cycling by wetlands and possible effects of water levels. Coastal Wetlands. Lewis Publishers, Chelsea Michigan, 1985: $69-86$.

[10] Pant H K, Reddy K R. Hydrologic influence on stability of organic phosphorus in wetland detritus. Journal of Environmental Quality, 2001, 30: 668 - 674.

[11] Richardson CJ. Mechanisms controlling phosphorus retention capacity in freshwater wetlands. Science, 1985, 228(4706) : $1424-1426$.

[12] Fisher M M, Reddy K R. Phosphorus flux from wetland soils affected by long-term nutrient loading. Journal of Environmental Quality, 2001, 30: $261-271$.

[13] Pant H K, Reddy K R. Potential internal loading of phosphorus in a wetland constructed in agricultural land. Water Research, 2003, 37 : 965 -972.

[14] Zhang Q, Volker R E. Lockington D A. Dispersion-based transport boundary condition in numerical modelling of density-dependent groundwater flow. Handbook and Proceedings of Water 99 Joint Congress, Brisbane, Australia, 6 -8 July 1999, Volume 2: 785 - 790 .

[15] Fetter CW. Contaminant Hydrogeology. USA: Macmillan Publishing Company, 1993.

[16]《水和废水监测分析方法》编委会. 水和废水监测分析方法, 第四版. 北京: 中国环境科学出版 社, 2002.

[17] Mitsch W J, Reeder B C. Nutrient and hydrologic budgets of a great lakes coastal freshwater wetland during a drought year. Wetlands Ecology and Management, 1992, 1(4) : $211-222$. 